\title{
Advanced numerical simulation and modelling for reactor safety - contributions from the CORTEX, HPMC, McSAFE and NURESAFE projects
}

\author{
Christophe Demazière ${ }^{1, *}$, Victor Hugo Sanchez-Espinoza ${ }^{2}$, and Bruno Chanaron ${ }^{3}$ \\ ${ }^{1}$ Department of Physic, Division of Subatomic and Plasma Physics, Chalmers University of Technology, 41296 Gothenburg, \\ Sweden \\ 2 Institute for Neutron Physics and Reactor Technology (INR), Karlsruhe Institute of Technology (KIT), \\ Hermann-vom-Helmholtz-Platz-1, 76344 Eggenstein-Leopoldshafen, Germany \\ ${ }^{3}$ Commissariat à l'Energie Atomique et aux Energies Alternatives, Centre de Saclay, 91191 Gif-sur-Yvette Cedex, France
}

Received: 12 March 2019 / Accepted: 4 June 2019

\begin{abstract}
Predictive modelling capabilities have long represented one of the pillars of reactor safety. In this paper, an account of some projects funded by the European Commission within the seventh Framework Program (HPMC and NURESAFE projects) and Horizon 2020 Program (CORTEX and McSAFE) is given. Such projects aim at, among others, developing improved solution strategies for the modelling of neutronics, thermal-hydraulics, and/or thermo-mechanics during normal operation, reactor transients and/or situations involving stationary perturbations. Although the different projects have different focus areas, they all capitalize on the most recent advancements in deterministic and probabilistic neutron transport, as well as in DNS, LES, CFD and macroscopic thermal-hydraulics modelling. The goal of the simulation strategies is to model complex multi-physics and multi-scale phenomena specific to nuclear reactors. The use of machine learning combined with such advanced simulation tools is also demonstrated to be capable of providing useful information for the detection of anomalies during operation.
\end{abstract}

\section{Introduction}

The safe and reliable operation of nuclear power plants relies on many intertwined aspects involving technological and human factors, as well as the relation between those. On the technological side, the pillars of reactor safety are based on the demonstration that a reactor can withstand the effect of disturbances or anomalies. This includes the prevention of incidents and should an accident occur, its mitigation.

Predictive simulations have always been one of the backbones of nuclear reactor safety. Due to the extensive efforts the Verification and Validation $(\mathrm{V} \& \mathrm{~V})$ of the corresponding modelling software these represent, most of the tools used by the industry are based on coarse mesh in space and low order in time approaches developed when computing resources and capabilities were limited. Because of the progress recently made in computer architectures, high performance computing techniques can be used for

\footnotetext{
* e-mail: demaz@chalmers.se
}

modelling nuclear reactor systems, thus replacing the legacy approaches by truly high-fidelity methods.

In parallel with the more faithful modelling of such systems, the monitoring of their instantaneous state is becoming increasingly important, so that possible anomalies can be detected early on and proper actions can be promptly taken. On one hand, over $60 \%$ of the current fleet of nuclear reactors is composed of units more than 30 years old, therefore, operational problems are expected to be more frequent. On the other hand, the conservatism in design previously applied to the evaluation of safety parameters has been greatly reduced, thanks to the increased level of fidelity achieved by the current modelling tools. As a result, nuclear reactors are now operating more closely to their safety limits. Operational problems may be also accentuated by other factors (e.g. use of advanced high-burnup fuel designs and heterogeneous core loadings).

In this paper, a brief account of four projects previously or currently funded by the European Commission in the area of the simulation and the monitoring of nuclear reactor systems is given. Despite the differences in nature between those projects, the key objectives and achievements with 
respect to advanced numerical simulation and modelling for reactor safety will be given particular emphasis. The paper will conclude with some recommendations for the future.

A glossary defining all the used abbreviations can be found at the end of the paper.

\section{Short description of the respective projects}

\subsection{CORTEX}

The CORTEX project (with CORTEX standing for CORe monitoring Techniques and EXperimental validation and demonstration) is a research and innovation action financed by the European Commission. The project formally started on September 1, 2017 for a duration of four years. The overall objective of CORTEX is to develop a core monitoring technique allowing the early detection, localization and characterization of anomalies in nuclear reactors while operating.

Being able to monitor the state of reactors while they are running at nominal conditions is extremely advantageous. The early detection of anomalies gives the possibility for the utilities to take proper actions before such problems lead to safety concerns or impact plant availability. The analysis of measured fluctuations of process parameters (primarily the neutron flux) around their mean values has the potential to provide non-intrusive online core monitoring capabilities. These fluctuations, often referred to as noise, primarily arise either from the turbulent character of the flow in the core, from coolant boiling (in the case of twophase systems), or from mechanical vibrations of reactor internals. Because such fluctuations carry valuable information concerning the dynamics of the reactor core, one can infer some information about the system state under certain conditions.

A promising but challenging application of core diagnostics thus consists in using the readings of the (usually very few) detectors (out-of-core neutron counters, in-core power/flux monitors, thermocouples, pressure transducers, etc.), located inside the core and/or at its periphery, to backtrack the nature and spatial distribution of the anomaly that gives rise to the recorded fluctuations.

Although intelligent signal processing techniques could also be of help for such a purpose, they would generally not be sufficient by themselves. Therefore, a more comprehensive solution strategy is adopted in CORTEX and relies on the determination of the reactor transfer function or Green's function, and on its subsequent inversion.

The Green's function establishes a relationship between any local perturbation and the corresponding spacedependent response of the neutron flux throughout the core. In CORTEX, state-of-the-art modelling techniques relying on both deterministic and probabilistic methods are being developed for estimating the reactor transfer function. Such techniques are also being validated in specifically designed experiments carried out in two research reactors.

Once the reactor transfer is known, artificial intelligence methods relying on machine learning techniques are used to recover from the measured detector signals the driving anomaly, its characteristic features and location.

More information about the CORTEX project can be found in [1].

\subsection{HPMC and McSAFE}

The projects HPMC (High Performance Monte Carlo Methods for Core Analysis) and McSAFE (High Performance Monte Carlo Methods for SAFEty Analysis) are two collaborative research projects funded by the European Commission in the seventh Framework Program (20112013) and Horizon 2020 Program (2017-2020) with the main goal of developing high fidelity multi-physics simulation tools for the improved design and safety evaluation of reactor cores. The peculiarity of HPMC and McSAFE is the focus on Monte Carlo neutronics solvers instead of deterministic ones, in order to take profit of the huge and cheap available computer power currently available.

The scientific goal of the HPMC was the "proof of concept" of newly developed multi-physics codes for depletion analysis taking into account thermal hydraulic feedbacks, static pin-by-pin full LWR core analysis considering local feedback, and the development of timedependent Monte Carlo codes including the behaviour of prompt and delayed neutrons for accident analysis.

Based on the success and promising results of the HPMC project, the goal of the McSAFE project that started in September 2017 is to become a powerful numerical tool for realistic core design, safety analysis and industry-like applications of LWRs of Generation II and III $[2,3]$. For this purpose, the envisaged developments will permit to predict important core safety parameters with less conservatism than current state-of-the-art methods and they will make it possible to increase the performance and operational flexibility of nuclear reactors. Moreover, the multi-physics coupling developments are carried out within the European Simulation platform NURESIM developed during different projects in the seventh Framework Program such as NURESIM, NURISP and NURESAFE [4], heavily relying on the open-source SALOME-software platform. In this context, the European Monte Carlo solvers MONK, SERPENT, and TRIPOLI are coupled with the subchannel thermal-hydraulic code SUBCHANFLOW and with the thermo-mechanic solvers TRANSURANUS using the ICoCo-methodology [5]. At present, the application and demonstration are done for LWRs and SMRs. However, the peculiarity of the codes and methods make their application possible to the Gen-III and Gen-IV reactors as well as to research reactors, for which the complicated geometry and physics of the core can only be adequately simulated by Monte Carlo codes.

Finally, all developed methods and codes are validated against plant data of European VVER and PWR plants as well as using test data of the SPERT Series IV E REA.

\subsection{NURESAFE}

NURESAFE (NUclear REactor SAFEty simulation platform) is a collaborative research project funded by the 
European Commission in the seventh Framework Program $[5,6]$. The project started early 2013 for a duration of three years. The main objective of NURESAFE was to develop a European reference tool for higher fidelity simulation of LWR cores for design and safety assessment.

The simulation tool developed by the NURESAFE project includes deterministic core physics codes, thermalhydraulics and fuel thermo-mechanics codes, all integrated in a software platform whose name is NURESIM. This platform provides a capability for code coupling, capability of paramount importance as the main phenomena occurring in reactors involve an interaction between the abovementioned physics. The NURESIM platform also offers an uncertainty quantification, which is necessary for validation and safety evaluation.

The scope of the NURESIM platform includes the simulation of steady states of LWRs and design basis accidents of LWRs. This platform was initially created in the framework of former collaborative projects within the sixth and seventh Framework Programs (NURESIM and NURISP), during which core physics and thermalhydraulics codes were first integrated. In NURESAFE, the platform was extended to more codes, particularly fuel thermo-mechanics codes. An important part of the NURESAFE work was also dedicated to:

- the demonstration of the multi-physics capability of the platform;

- advanced CFD modelling;

- uncertainty quantification and validation.

\section{Key objectives with respect to advanced numerical simulation and modelling for reactor safety}

\subsection{Introduction}

As earlier mentioned, most of the modelling tools used by the nuclear industry were developed when computing resources and capabilities were limited. Although nuclear reactors are by essence multi-physics and multi-scale systems, the techniques that were then favoured relied on modelling the different fields of physics and sometimes the different scales by different codes that were only thereafter coupled between each other. In the current best-estimate approaches, the modelling of neutron transport, fluid dynamics and heat transfer is thus based on a multi-stage computational procedure involving many approximations.

On the neutronic side, deterministic approaches have been used primarily, due to their lower computational cost compared to probabilistic methods (i.e. Monte Carlo). Deterministic tools nevertheless rely on many approximations, with the neutron transport equation solved explicitly after reducing the complexity of the task at hand (typically using space-homogenization, energy-condensation, and angular approximation techniques) [7]. The problem is first solved over a small region of the computational domain using approximate boundary conditions, and the "fine-grid" solution then computed is used for producing equivalent average properties locally. In a second step, a global "coarse-grid" solution is found for the full computa- tional domain, in which only average local properties are considered, i.e., in which the true complexity of the system is not represented explicitly. Typically, three to four of such "bottom-up" simplifications are used to model a full reactor core. Although used on a routine basis for reactor calculations, the approximations used in each of the computational steps are almost never corrected by the results of the calculations performed in the following steps when a "better" (i.e. taking a larger computational domain into account) solution has been computed.

In the probabilistic approach on the other hand, no equation as such is solved. Rather, the probability of occurrence of a nuclear reaction/process of a given type on a given nuclide at a given energy for a given incoming particle (which can still exist after the nuclear interaction) is used to sample neutron life histories throughout the system [8]. Using a very large number of such histories, actual neutron transport in the system can be simulated without requiring any simplification, and statistically meaningful results can be derived by appropriately averaging neutron tallies. However, due to the size and complexity of the systems usually modelled, Monte Carlo techniques are extremely expensive computing techniques, which limited their use for routine applications in the past.

With the advent of cheap computing resources, both the deterministic approach and the probabilistic approach are now being used on massively parallel clusters to circumvent the limitations mentioned above. In the deterministic case, the process of averaging ("bottomup") is now being complemented by a de-averaging process ("top-down") in an iterative manner, so that a better modelling of the boundary conditions can be achieved using the information available from the coarser mesh. The modelling of full cores in a single computational step is also being contemplated. In the probabilistic case, the use of large clusters allows modelling full reactor cores, and efforts are being pursued to include the feedback effects induced by changes in the composition and/or density of the materials $[9,10]$. Due to the complexity and level of details in the deterministic approach based on the averaging/ de-averaging process, there are situations where the deterministic route can become quite expensive, being almost on par with the probabilistic route for high-fidelity simulations.

On the thermal-hydraulic side, the strategy is to average in time and in space the local conservation equations expressing the conservation of mass, momentum and energy. The double averaging results in a set of macroscopic conservation equations that are tractable for a large system as a nuclear reactor, unfortunately at the expense of filtering the high-frequency and small-scale phenomena [7]. In addition, the averaging process introduces new unknown quantities (expressing for instance the wall transfer and possible interfacial transfer between the phases) that are usually determined using empirical or semi-empirical correlations. These correlations are heavily dependent on the flow regimes. Such a modelling strategy is often referred to as a system code approach. With the advent of cheap computing power, current efforts focus on modelling much finer scale using CFD tools instead. 


\subsection{CORTEX}

For the CORTEX project, since a majority of the diagnostic tasks are based on the inversion of the Green's function, the key objectives in the area of advanced numerical simulation and modelling can be summarized as follows: (a) the development of modelling capabilities for estimating the transfer function, (b) the validation of such tools against experiments specifically designed for that purpose, and (c) the inversion of the reactor transfer function using machine learning.

Concerning (a), one of the strategic objectives of the project is to determine the area of applicability of existing tools for noise analysis and to develop new simulation tools that are specifically dedicated to the modelling of the effect of stationary fluctuations in power reactors with a high level of fidelity. The ultimate goal is to develop modelling capabilities allowing the determination, for any reactor core, of the fluctuations in neutron flux resulting from known perturbations applied to the system. Two tracks are followed. Existing low-order computational capabilities are consolidated and extended. Simultaneously, advanced methods based on deterministic neutron transport and on probabilistic (i.e. Monte Carlo) methods are developed so that the transfer function of a reactor core can be estimated with a high resolution in space, angle and energy. Since the modelling of the response of the system to a perturbation expressed in terms of macroscopic cross-sections is equally important as the modelling of the actual perturbation, large efforts are spent on converting actual noise sources into perturbations of cross-sections. For that purpose, emphasis is put on developing models for reproducing vibrations of reactor vessel internals due to FSI. Finally, the evaluation of the uncertainties associated to the estimation of the reactor transfer function is given particular attention, together with the sensitivity of the simulations to input parameters and models.

Concerning (b), although the tools allowing estimating the reactor transfer function can be verified against analytical or semi-analytical solutions for simple systems and configurations, the validation using reactor experiments specifically designed for noise analysis applications is essential. Two types of neutron noise measurements are considered: a so-called absorber of variable strength and a so-called vibrating absorber.

Finally, concerning (c), the backtracking of the driving perturbation (not measurable) from the induced neutron noise (measurable at some discrete locations throughout the core) is performed using machine learning. With the tools referred to above, the induced neutron noise for many possible scenarios of considered perturbations is estimated. The results of such simulations are then provided as training data sets to machine learning techniques. Based on such training sets, the machine learning algorithms have for primary objective to identify the scenario existing in a nuclear core from the neutron noise recorded by the in- and ex-core neutron detectors and, when relevant, retrieve the actual perturbation (and its location).

\subsection{HPMC and McSAFE}

The major objectives of the HPMC project were the following:

(a) optimal Monte Carlo-thermal-hydraulics coupling: the objective was to realise efficient coupling of the Monte Carlo codes SERPENT and MCNP with the thermalhydraulic subchannel codes SUBCHANFLOW and FLICA4, suitable for full core applications;

(b) optimal Monte Carlo burn-up integration: the objective was to realise an efficient integration of burnup calculations in the Monte Carlo codes SERPENT and MCNP, suitable for full core applications;

(c) time-dependence capabilities in Monte Carlo methods: the objective was to develop an efficient algorithm for modelling time-dependence in the Monte Carlo codes SERPENT and MCNP, applicable to safety analysis and full core calculations.

Based on the promising results of the HPMC project, the McSAFE project started in September 2017 with the goal to move the Monte Carlo-based multi-physics codes towards industrial applications, e.g. simulation of depletion of commercial LWR cores taking thermal-hydraulic feedback into account, analysis of transients such as REA. For this purpose, a generic and optimal coupling approach based on ICoCo and the open-source NURESIM platform is followed for the coupling of the European Monte Carlo solvers such as MONK, SERPENT and TRIPOLI with subchannel codes, e.g. SUBCHANFLOW and fuel thermo-mechanics solvers, e.g. TRANSURANUS. Moreover, dynamic versions of TRIPOLI, SERPENT and MCNP6 coupled with SUBCHANFLOW are developed for analysing transients. Especially, SERPENT/SUBCHANFLOW is being coupled with TRANSURANUS for the depletion analysis of commercial western PWR and VVER cores while considering thermal-hydraulic feedback. Emphasis is put on the extensive validation of the tools being developed within McSAFE. For the validation of the depletion capabilities, plant data are used, whereas for the validation of the dynamic capability of the coupled Monte Carlo-thermal-hydraulics codes under development, experimental data of unique tests e.g. the SPERT REA IV E are used. Finally, high fidelity tools based on Monte Carlo requires a massive use of HPC in order to solve full cores at the pin level. Methods for optimal parallelization strategy, scalability of Monte Carlo-based simulations of depletion problems and time-dependent simulations, are also scrutinized in the McSAFE project. Since memory requirements for such problems may represent a limiting factor, methods for the optimal use of memory during depletion simulations of large problems needs to be further developed.

\subsection{NURESAFE}

The main objectives of NURESAFE were as follows:

- To enhance the prediction capability of the computations used for safety demonstration of the current LWR nuclear power plants through the dynamic $3 \mathrm{D}$ coupling of 
the codes, simulating the different physics of the problem into a common multi-physics simulation scheme.

- To advance the fundamental knowledge in two-phase thermal-hydraulics and develop new multi-scale thermalhydraulics models. Emphasis was put on coupling interface tracking models with phase-averaged models. Moreover, pool and convective boiling were given special attention, together with the physics of bubbly flow.

- To develop multi-scale and multi-physics simulation capabilities for LOCA, PTS and BWR thermal-hydraulics, thus allowing more accurate and more reliable safety analyses. The aim was to develop a European reference tool for higher fidelity simulation of LWR cores for design and safety assessments. The delivery of safety-relevant industry-like applications was also one of the primary objectives of the project, so that the various applications could be used by the industry at the completion of the project.

- To develop generic software tools within the NURESIM software platform and to provide a support to developers for integration of the codes into this platform.

\section{Key achievements with respect to advanced numerical simulation and modelling for reactor safety}

\subsection{CORTEX}

Since the start of the project, the key achievements in the area of advanced numerical simulation and modelling along the three objectives identified in Section 3.2 can be summarized as follows.

\subsubsection{Development of modelling capabilities for estimating the transfer function}

The work carried out so far is performed along several lines.

In the area of mechanical vibrations, an extensive review of the past work on vibration of reactor internals was carried out. The focus was on both obtaining a coverage of all possible sources of neutron noise, a phenomenological description of each corresponding scenario, and of the observed neutron noise patterns when actual plant measurements were available. First simulations using thermal-hydraulic perturbations generated by a system code were later fed into a FEM code modelling mechanical structures.

In parallel to those activities, neutronic capabilities are being developed. For coarse mesh approaches, three parallel tracks are pursued. Nodal codes used for the simulation of other core transients in the time-domain are used. To use some of these codes, the first step is to generate a set of time-dependent macroscopic cross-sections that simulate the movement of the fuel assemblies on a fixed computational coarse grid, based on the results of the FSI simulations. Procedure are being implemented to generate the whole set of cross sections. In addition to the use of existing time-dependent tools with a set of time-dependent cross sections, another approach is pursued based on the development of an ad-hoc software relying on FEM. The FEM method has a large versatility for solving balance equation using different spatial meshes and a code is being developed along those lines. It will offer the possibility in the future to have a moving mesh following the vibration characteristics determined from the FSI calculations. The main advantage of the FEM route lies with the fact that only static macroscopic cross sections for the initial configuration of the core are necessary. Finally, a third and complementary approach based on a mesh refinement technique in the frequency domain is being developed. The modelling of vibrating reactor internals requires the definition of perturbations on very small spatial domains compared to the size of the node size used in coarse mesh modelling tools. This makes it necessary to develop mesh refinement techniques around the region where the perturbation exists. This mesh refinement technique is currently implemented in a frequency-domain core simulator earlier developed. For fine mesh approaches, deterministic methods relying on the method of discrete ordinates (Sn) are being developed. Moreover, a neutron noise solver relying on the method of characteristics is being implemented. In probabilistic methods, an equivalence procedure between neutron noise problems in the frequency-domain and static subcritical systems is being developed. A method using complex statistical weights and a modified collision kernel for the neutron transport equations in the frequency domain have been implemented in a Monte-Carlo code. Likewise, another method using complex-valued weights in the frequency domain has been implemented.

As can be seen above, several complementary approaches are being developed. They either rely on existing codes or codes specifically developed for noise analysis. Moreover, these codes work either in the time or in the frequency domain. These tools use either a coarse-mesh approach (possibly with a moving mesh) or a fine-mesh approach regarding the spatial discretization. Finally, both deterministic and probabilistic methods are considered.

\subsubsection{Validation of the modelling capabilities against experiments}

Concerning the validation of such tools against experiments specifically designed for neutron noise, two research facilities are used: the AKR-2 facility at TUD, Dresden, Germany, and the CROCUS facility at EPFL, Lausanne, Switzerland. Pictures of those two facilities are given in Figure 1.

The perturbation was simultaneously recorded by 7 and 11 neutron detectors, for the first AKR-2 and CROCUS campaigns, respectively, located throughout the respective cores, together with the recording of the actual perturbation introduced. The data acquisition systems were successfully benchmarked against an industry-grade data acquisition system from TUV Rheinland ISTec GmbH. In terms of perturbations, AKR-2 has the ability to perturb the system in two ways: either by rotating a neutron 


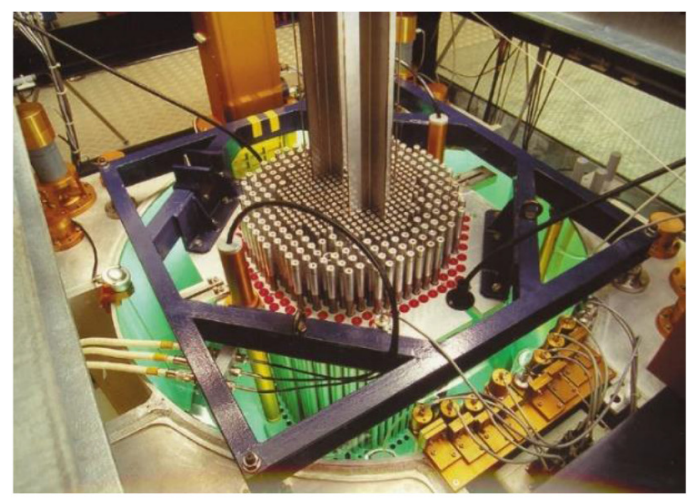

(a) CROCUS (courtesy of EPFL)

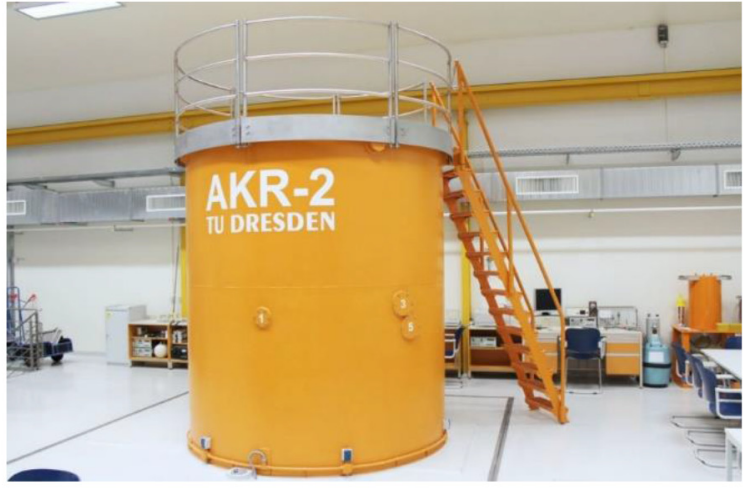

(b) AKR-2 (courtesy of TUD)

Fig. 1. Overview of the CROCUS and AKR-2 facilities.

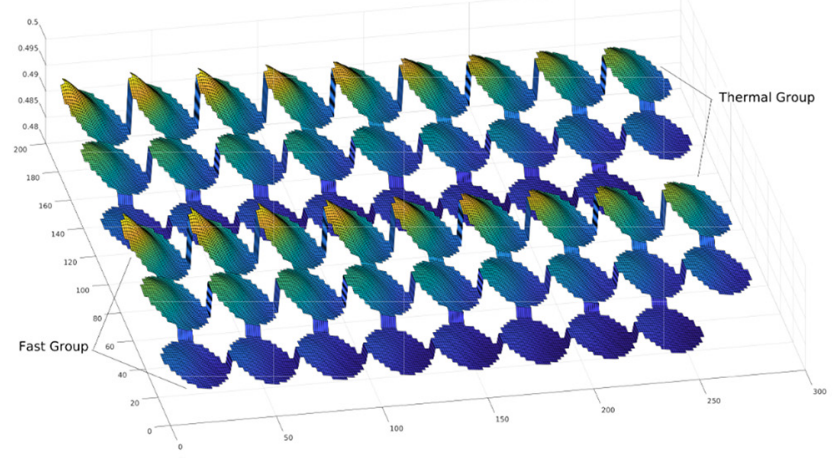

(a) Phase information

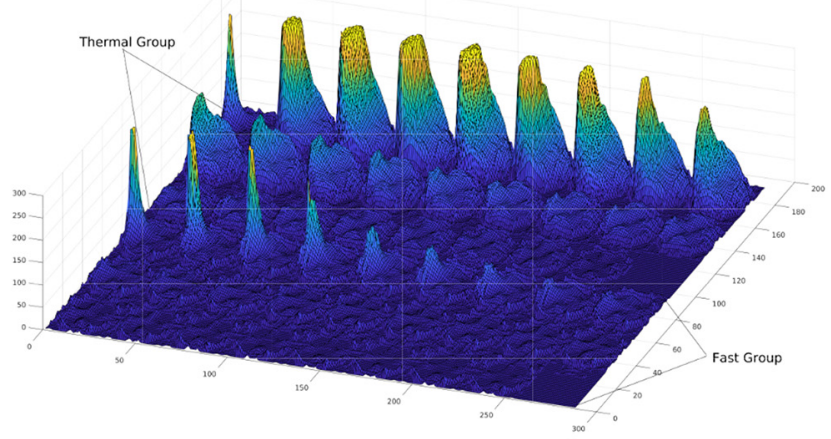

(b) Amplitude information

Fig. 2. Example of the reactor response to a localized absorber of variable strength unrolled as two-dimensional images (courtesy of University of Lincoln) [11].

absorbing foil (thickness of $0.02 \mathrm{~cm} \times$ length of $25 \mathrm{~cm} \times$ width of $2 \mathrm{~cm}$ ) along a horizontal axis or by moving a neutron absorbing disc (thickness of $1.0 \mathrm{~mm} \times$ diameter of $12.7 \mathrm{~mm}$ ) along a horizontal axis. In the former case, the foil rotates at a distance of $2.98 \mathrm{~cm}$ from its axis at a frequency of up to $2.0 \mathrm{~Hz}$, whereas in the latter case, the disc is moving horizontally with a maximum displacement amplitude of $20 \mathrm{~cm}$ at a frequency up to $2.0 \mathrm{~Hz}$. At CROCUS, up to 18 fuel rods located at the periphery of the core can be displaced laterally with a maximum displacement up to $\pm 2.5 \mathrm{~mm}$ from their equilibrium positions at a frequency up to $2 \mathrm{~Hz}$. The first noise measurements for the three types of noise sources (rotating absorber and vibrating absorber at AKR-2; vibrating fuel rods at CROCUS) have been performed as part of the validation of the data acquisition systems.

Since both the perturbations and the corresponding induced neutron noise are recorded in the experiments described above, such experiments can be used to validate the neutronic tools aimed at estimating the Green's function of the reactor and being developed within
CORTEX. Such noise measurements, where both the perturbations and the corresponding neutron noise are recorded, represent a world premiere.

\subsubsection{Inversion of the reactor transfer function using machine learning}

Preliminary tests were performed using simulated signals, either in the time domain or in the frequency domain. Several scenarios corresponding to different types of noise sources were considered: localized absorbers of variable strength in the frequency domain, travelling perturbations along fuel channels in the frequency domain, fuel assembly vibrations in the time domain, and inlet coolant perturbations in the time domain. First successful machine learning tests on the absorbers of variable strength were based on "unrolling" the three-dimensional induced neutron noise into the juxtaposition of two-dimensional images, each corresponding to the plane-wise response of the reactor core to the perturbation [11]. Figure 2 represents such twodimensional information that was then fed to a Deep CNN 


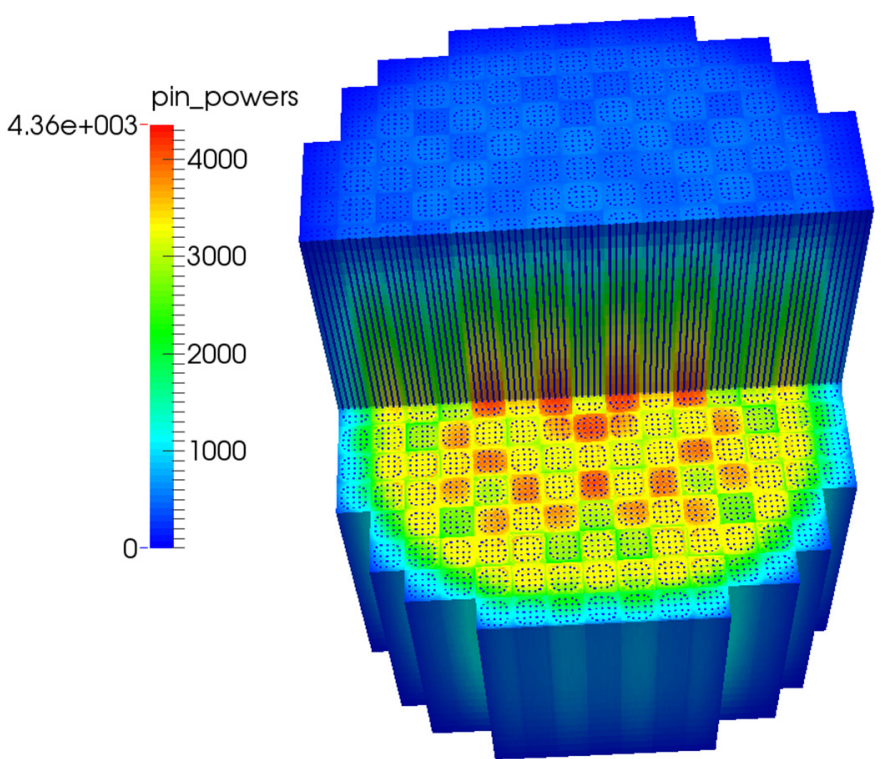

Fig. 3. 3D pin power predicted by SERPENT/SUBCHANFLOW for the PWR UOX/MOX core [10].

to retrieve the actual location of the perturbation. The recovery of the exact spatial location of the noise source was thereafter improved by using instead a three-dimensional CNN, so that the axial coupling information could be fully exploited in the unfolding [12]. In addition, both the absorber of variable strength data and the travelling perturbation data were used. The network could both recognize the type of perturbation applied and recover the actual location of the perturbation being applied. For the time-domain data, the different scenarios could be successfully identified using a LSTM network.

\subsection{HPMC and McSAFE}

\subsubsection{Optimal Monte Carlo-thermal-hydraulics coupling}

The HPMC project demonstrated the potentials and capabilities of Monte Carlo-based multi-physics coupled codes for improved static core analysis taking local interdependencies between neutronics and thermalhydraulics into account. At the completion of the project, two coupled codes, SERPENT/SUBCHANFLOW and MCNP/SUBCHANFLOW, had been developed for static full core simulations at the pin level. Those codes were successfully applied to the analysis of a PWR core with UOX and MOX fuel assemblies, while taking local thermalhydraulic feedback into account and using HPC clusters $[9,10]$. As an illustrative example, the capability of the coupled code SERPENT/SUBCHANFLOW to perform a pin-level analysis of a full PWR core with local thermalhydraulic feedback is shown in Figure 3. The problem consists of 55777 neutronic nodes (pins and guide tubes), 2.2 million fluid cells, as well as 23.4 million solid cells (thermal-hydraulic solver). A total of $4 \times 10^{6}$ neutrons per cycle and 650 inactive and 2500 active cycles were used in the SERPENT calculations. The simulation was performed at the KIT IC2 HPC cluster using 2048 cores. A converged solution was achieved after 5.8 CPU-year (1.03 days).

\subsubsection{Optimum Monte Carlo burn-up integration}

Another important outcome was the exploration and development of various schemes for stable depletion calculation using Monte Carlo codes such as the SIE method [13] for stable steady-state coupled Monte Carlothermal-hydraulics calculations.

\subsubsection{Time-dependence capabilities in Monte Carlo methods}

A highlight of the project was the implementation of a time-dependence option in MCNP5 (dynMCNP) that required source code modifications [14]. This option includes the generation and decay of delayed neutron precursors, possible control rods movement, etc. To reduce the statistical error in the generated reactor power in successive time intervals, a method of forced decay of precursors in each time interval was implemented. Moreover, variance reduction methods (like the branchless collision method) were introduced. Thermal-hydraulic feedback was also implemented. To let the time-dependent thermal-hydraulic calculations take the heating history into account, further extensions of the codes were necessary.

Finally, various ways for parallel execution of a Monte Carlo calculation using the MPI and OpenMP application programming interfaces were investigated and their efficiency measured in terms of the speedup factor. For application on large computer clusters with different computer nodes and multiple processors per node, the optimum combination of MPI and OpenMP was determined. Application of OpenMP was introduced in the SERPENT2 code. The MCNP code was modified to use all available processor cores for neutron history simulation [15].

The main achievements close to the midterm of the McSAFE-project are described hereafter.

Full core multi-physics depletion: Methods for depletion of full core using Monte Carlo codes are being developed. First of all, the efficiency and stability of Monte Carlo burnup simulations were studied by optimal combination of free parameters that allow solving full core problems [16]. In addition, a collision-based domain decomposition scheme for SERPENT2 is being developed to solve large-scale high-fidelity problems with large memory demands (e.g. full core pin-by-pin depletion). For this purpose, memory-intensive materials are split among MPI tasks, enabling the memory demand to be divided among nodes in a high-performance computer [17]. Investigations were also performed to identify the computational requirements for depletion calculations taking thermal-hydraulic feedback into account for $3 \mathrm{D}$ problems (e.g. $5 \times 5$ fuel assemblies mini-core) [18]. Potential bottlenecks and limitations, e.g. huge RAMrequirements which increase linearly with the number of fuel assemblies - 40 GB for eight fuel assemblies - could be identified. Alternatives were also proposed to overcome the challenges, such as a collision-based domain decomposition.

Code integration: The European Monte Carlo codes TRIPOLI, SERPENT, and MONK as well as the fuel 


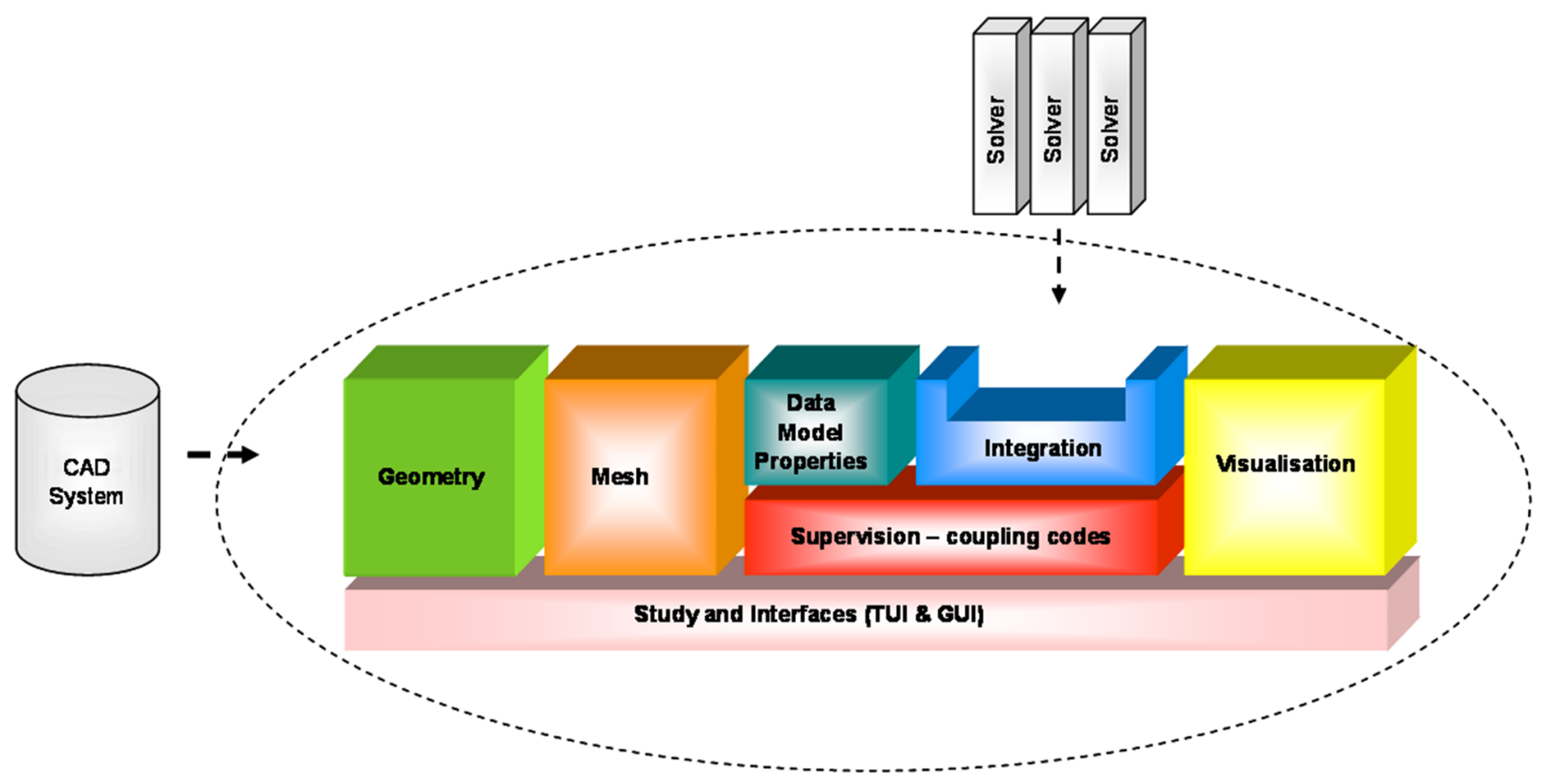

Fig. 4. SALOME global view.

thermo-mechanics code TRANSURANUS were fully integrated into the European NURESIM simulation platform (SUBCHANFLOW - SCF was already part of the platform). Each solver owns a specific meshing. New flexible and object-oriented coupling schemes based on the ICoCo-methodology are being developed for each of the codes integrated into the NURESIM platform. The following coupled code versions are available: MONK/ SCF, SERPENT/SCF, TRIPOLI/SCF.

Dynamical multi-physics calculations: Another important task in the McSAFE project is to extend generalpurpose Monte Carlo codes (SERPENT2, TRIPOLI-4 and MCNP6) to dynamic version that can accurately calculate transient behaviour in nuclear reactors considering local thermal-hydraulic feedback. New versions of Monte Carlo codes with time-dependent capabilities (called dynamicMC) are at the end of the development phase for the analysis of transients. These Monte Carlo codes are coupled with the SCF thermal-hydraulic solver, thus leading to the coupled codes: dynMCNP/SCF, dynTRIPOLI/SCF, dynSERPENT/SCF. The code extensions and modifications are described in more detail in $[14,19,20]$. The coupling schemes must be appropriate for massive HPC-simulations. The peculiarity of time-dependent Monte Carlo is to describe the behaviour of delayed neutrons, which have a significant influence on the statistical uncertainty (standard deviation) of the power prediction. An additional challenge is the short lifetime of prompt neutrons (roughly $100 \mu \mathrm{s}$ in an LWR) compared to the large decay time of precursors of delayed neutrons for the method development. To test the dynamic capability of the Monte Carlo codes, different REA scenarios are being developed within McSAFE.

\subsection{NURESAFE}

\subsubsection{Simulation platform}

One of the main outcomes of the NURESIM and NURISP projects was the release of the NURESIM platform that is heavily used in NURESAFE. The NURESIM platform is based upon the software simulation platform SALOME. SALOME is an open-source project, (http://salomeplatform.org), which implements the interoperability between a CAD modeller, meshing algorithms, visualisation modules and computing codes and solvers, as represented in Figure 4. It mutualises a pool of generic tools for pre-processing, post-processing and code coupling. Its supervision module provides functionalities for code integration, dynamic loading and execution of components on remote distributed computing systems, and supervision of the calculation. Support is provided to developers for integration of the codes into the SALOME software and for producing and managing the successive versions of the NURESIM platform on a dedicated repository. Innovative deterministic and statistical methods and tools for quantification of the uncertainties developed within NURESAFE give a better knowledge of conservatisms and margins.

The NURESIM platform provides a set of state-of-theart software devoted to the simulation of normal operation and design basis accidents of LWR (i.e. BWR, PWR, and VVER). The platform includes 14 codes covering different physics: neutronics, thermal-hydraulics, fuel thermo-mechanics at different scales, 2 thermal-hydraulics system codes, 2 single-phase CFD codes, 2 two-phase CFD codes, 3 sub-channel thermal-hydraulic analysis codes, 2 advanced fuel thermo-mechanics codes, 2 DNS codes, 3 neutron- 

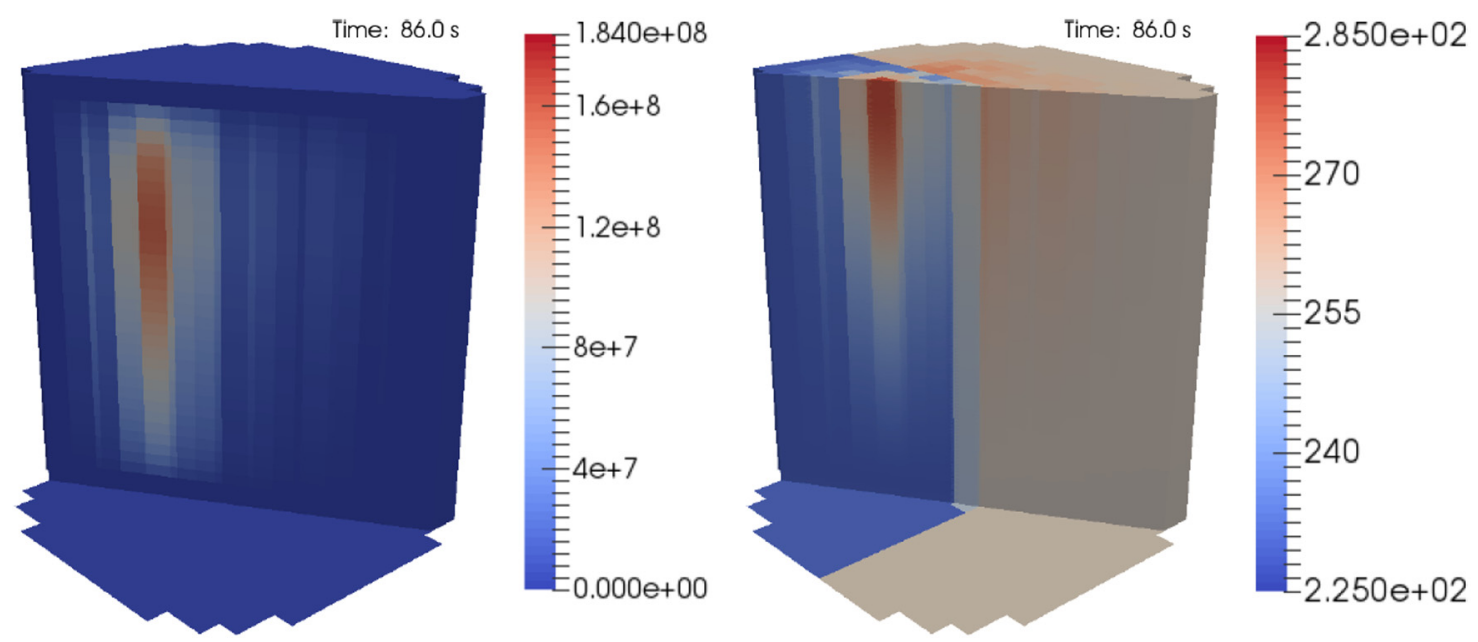

Fig. 5. Distribution of power density $\left(\mathrm{MW} / \mathrm{m}^{3}\right.$, left $)$ and coolant temperature $\left({ }^{\circ} \mathrm{C}\right.$, right $)$ at $86 \mathrm{~s}$ after the initiation of the $\mathrm{MSLB}$ event.

kinetics codes. All these codes were extensively benchmarked and validated against experiments during the course of the NURESAFE project.

SALOME is connected to URANIE, an open-source platform aimed at providing methods and algorithms about uncertainty and sensitivity, and verification and validation analyses in the same framework (https:// sourceforge.net/projects/uranie/). The URANIE and SALOME platforms work nicely together. Any calculation scheme developed in SALOME can be used within URANIE.

Through the link with URANIE, users of the NURESIM platform successfully performed in the NURESAFE project sensitivity analyses and model calibration studies.

\subsubsection{D dynamic coupling of codes}

Individual models, solvers, codes and coupled applications were run and validated through modelling "situation targets" corresponding to given nuclear reactor situations and including reference calculations, experiments, and plant data. As safety analysis was the main issue within the project, all these situation targets consisted in some accidental scenarios. The challenging "situation targets" were selected according to the required coupling between two different disciplines. Industry-like applications were released at the end of the project for the following "situation targets":

- Square lattice PWR MSLB;

- One selected BWR ATWS;

- VVER MSLB.

The analysis also included uncertainty quantification using the URANIE open-source software.

The BWR ATWS analysis framework featured coupled simulations combining system thermo-hydraulics, 3D neutronics, thermo-mechanical evaluation of fuel safety parameters, and uncertainty evaluation. The MSLB transient analysis provided more accurate assessment of margins between predicted key parameters and safety criteria. The outcome of the transient simulation was evaluated with respect to local re-criticality and maximum reactor power level. As an illustrative example, the results of the PWR MSLB are presented hereafter.

A two-step modelling approach was applied. In the first step, reference results were produced using the platform codes with higher resolutions of coupling between core nodal and sub-channel scale. In the second step, CFD evaluations were included into the solution. In that way, an improvement in the prediction of the target safety parameters could be achieved. In order to increase the confidence of the CFD results, a validation was also performed by comparing the calculation results with experimental data from the HZDR test facility on coolant mixing ROCOM. The cross-section libraries were created using new methods of grid point selection [21]. Various combinations of system codes, core thermal-hydraulic codes and neutronic codes were used. Figure 5 highlights the $3 \mathrm{D}$ distributions at time $t=86 \mathrm{~s}$ after the initiation of the MSLB.

The obtained results confirmed that the NURESIM platform is applicable for challenging coupled transients in PWRs. Furthermore, by accomplishing the coupling of reactor dynamics codes and CFD codes, the superiority of the NURESIM platform was demonstrated. The conducted advanced calculations demonstrated the excellent status and the readiness for industrial applications of the NURESIM platform and the integrated codes.

\subsubsection{Advanced CFD modelling}

Advancement in the fundamental knowledge of CFD modelling was pursued and new models based on detailed DNS for momentum exchange and boiling heat transfer situations typical of LWR thermal-hydraulics were developed. New benchmark data bases for fundamental and applied problems were developed. The existing computational multiphase flow strategies were first extended in order to cope with a wider range of practical applications. 
Novel methods for pool and convective boiling in a channel were also developed. Advanced strategies for modelling turbulent bubbly flow in a channel and in a rod bundle were analysed. Finally, the novel models and simulation techniques were implemented in codes, validated and applied in this context. New versions of the CFD platform codes NEPTUNE_CFD, TransAT and TRIO_U were delivered to end users, including the most advanced numerical simulation features and the associated modelling approaches for the physics pertinent to both PWRs and BWRs.

Three specific issues were addressed within NURESAFE: - All-topology flow modelling by coupling interface tracking models with phase-averaged models.

- DNS and LES of pool and convective boiling [22].

- DNS and LES of bubbly flows [23,24].

\subsubsection{Multi-scale and multi-physics simulations}

In the area of multi-scale and multi-physics simulations of LOCA, PTS and BWR thermal-hydraulics, multi-scale and multi-physics simulation capabilities for more accurate and more reliable safety analyses were developed.

LOCA is usually simulated with industrial versions of thermal-hydraulic system codes. Although system codes are able to address most safety needs, the status and limits of the current methods and tools for plant analysis were reviewed during the NURISP project and areas for improvements were pointed out. Advanced tools and methods for multi-scale and multi-physics analyses and simulations of LOCA, including situations with deformed or ballooned rods and possible fuel relocation, were developed. The addition to system thermal-hydraulic codes of two-phase CFD tools and of advanced fuel models allowed revisiting these transients for more accurate and reliable predictions. This required improving and coupling CFD to system codes or improving system codes and system codes coupled with fuel thermo-mechanics codes. Furthermore, methods for uncertainty and sensitivity analysis applied to system codes were improved. In this framework, a special focus was put on the issue of the quantification of the uncertainties of the closure laws. This work was based on a benchmarking of the possible methods using reflooding experimental data (FEBA and PERICLES).

Concerning PTS, better simulation capabilities were achieved by improving the CFD modelling thanks to the analysis of new experimental data (including TOPFLOW steam-water tests and KAERI CCSF test). In addition, sensitivity and uncertainty methods were applied to CFD codes and state-of-the-art methods on validation, uncertainty and sensitivity of CFD applications to reactor issues were reviewed.

In the field of BWR thermal-hydraulics, progress in the simulation of two-phase thermal-hydraulics phenomena specific to BWR was achieved. This includes dry-out prediction, transient core thermal-hydraulics and steam injection in pressure suppression pool. CFD codes and subchannel codes were used, improved and validated during the project.

\section{Training, education and dissemination activities}

\subsection{CORTEX}

The dissemination of the project results is carried out along five parallel lines of actions: involvement of end users into the project, organization of workshops, organization of short-courses, peer-reviewed publications, and presentations at conferences and meetings.

Concerning the involvement of end users, the project involves, beyond academic partners, research institutes, TSOs, utilities, fuel and reactor manufacturers, as well as services companies. Those organizations are either directly contributing to the project as project partners or participating to the project via the Advisory End User Group, having a consultative role to the consortium.

Three workshops will be organized:

- two workshops on the experiments performed at the research reactors and on the validation of the neutronic models based on such experiments, where experimentalists and modellers will present, describe and discuss their results;

- one (final) workshop on the demonstration of the methods developed within the project on actual plant data. During this workshop, the entire consortium will: (a) summarize the findings and the lessons learnt throughout the project, (b) give recommendations on techniques and instrumentations for core monitoring and surveillance (in order to improve the reliability and safety of the nuclear units); and (c) provide an outlook for the future in this area.

Eight short courses were/will be developed:

- two courses on reactor dynamics and neutron noise. Both courses were already given and had 47 registered participants in total. The first course covered the fundamentals of reactor kinetics and the theory of small space-time dependent fluctuations. The second course dealt with additional aspects, such as core thermalhydraulics, its coupling to neutron kinetics and reactor stability, and included hands-on training on the AKR-2 reactor at TUD;

- two courses/workshops on signal processing methods and their applications. Both courses/workshops were already arranged and attracted 64 attendees. The first course was an introduction to basic techniques for signal analysis and their possible applications. The second course dealt with advanced signal processing methods and statistical characterization of plant measurements, which can be applied to reactor core monitoring and dynamic sensor surveillance;

- one hands-on training session on the simulation of reactor neutron noise in power reactors using a time-domain neutron kinetics code. The students will have the opportunity to model different types of disturbances, such as fuel assembly vibrations, inlet disturbances, flow fluctuations, etc. and study their effect on the neutron flux throughout the entire system;

- one course on uncertainty and sensitivity analysis. Emphasis will be put on the application of such methods 
to the estimation of the reactor transfer function and the corresponding neutron noise;

- two hands-on training sessions on the two research facilities used in the project. The sessions will consist of the following exercises: reactor start-up procedures, control rod and critical experiments, and a set of neutron noise experiments.

In the area of publications, after 18 months as a running project, the following has been achieved:

- one journal publication (two more under review);

- eight conference publications (ten more under review);

- seven conference presentations.

In addition, most of the deliverables (26 in total - ten were already delivered) are/will be publicly available.

All the publicly available resources are directly accessible on the project website http://cortex-h2020.eu. In addition to the publications and deliverables listed above, newsletters are distributed once a year. The consortium is also heavily using LinkedIn http://link edin.com/company/cortex-h2020 to inform about the project. Promotional materials (video, leaflet, poster) are also available.

\subsection{HPMC and McSAFE}

The dissemination, education and training activities of both projects rely on the following pillars:

- dissemination plan for the identification of end users and stakeholders (industry, academia, regulators, TSO);

- creation of a public website http://www.mcsafe-h2020. eu;

- organisation of a dedicated training course to be held in April 2020 where the main tools of McSAFE will be presented and demos of selected applications will be shown to the community;

- presentation of the main results at international conferences, e.g. PHYSOR, M\&C, etc., publication of the main results in scientific journals, presentation at the NUGENIA Forum, the FISA Conference, etc;

- establishment of a Users' Group consisting of institutions which will get access to the use of the codes being developed and extended within McSAFE, for performing simulations of own problems. Important feedback from the Users' Group is expected regarding the capabilities and user-friendliness of the codes;

- creation of a Technical Advisory Board consisting of selected experts of the community of stakeholders and aimed at reviewing the McSAFE developments and at providing advice and comments on the main developments;

- delivery of 57 deliverables in total, from which around 30 are already finalized. Some of them are publicly available on the project website;

- education and training of young scientists through doctoral programs and through the involvement of master and bachelor students in the project at the different partner institutions.

\subsection{NURESAFE}

In order to foster the dissemination and facilitate the use of the platform codes, 15 training sessions of a few days each were given to the staff of the NURESAFE partners and to external users' organisations during the course of the project. The end users of the NURESIM platform and of the individual codes could thereafter efficiently use the tools and methods.

Two public NURESAFE general workshops were held in Budapest on June 16-17, 2014 and in Brussels on November 4-5, 2015, respectively, in order to present the new methods, models and functionalities that were developed. About 50 people attended each of the workshops.

Many publications were made:

- 12 articles were published in peer-reviewed journals (Annals of Nuclear Energy, International Journal of Heat and Fluid Flow, Multiphase Science and Technology, Nuclear Engineering and Design).

- 28 presentations were delivered at international conferences (NURETH, ICONE, CFD4NRS, SNA$\mathrm{M \& C}, \ldots)$.

An active Users' Group was set up when starting the project. The objective was to give the opportunity to organizations which were not members of the NURESAFE consortium to use and test the new methods and tools. Five universities and companies were members of the NURESAFE Users' Group: 3 non-European and 2 European. They provided fruitful feedback on the use of the codes in some challenging situations, especially in thermal-hydraulics.

\section{Utilization and cross-fertilization}

CORTEX is by essence an international project, since one of the partners is from USA and another one is from Japan. Moreover, the project gathers academic partners, research institutes, TSOs, utilities, fuel and reactor manufacturers, as well as services companies in order to develop a core monitoring technique in close dialogue with all relevant stakeholders. This will result in a method directly applicable for the industry. Finally, additional interest was received from the USA for developing a similar method as the one being developed in CORTEX.

Although neutron noise core monitoring has been used in a "rudimentary" manner in some plants worldwide, the methodology proposed in CORTEX and relying on machine learning techniques combined with dedicated neutron noise simulations has never been attempted. Moreover, the development of neutron noise simulation capabilities at an industrial level also represents a novelty in CORTEX. Being able to infer from the detector readings the existence, location and features of possible anomalies would represent a world-premiere.

If successful, the project will also be able to identify the root-cause of some operational problems during exploitation. CORTEX will for instance investigate the increase of the neutron noise levels observed in some Pre-KONVOI 
PWRs, events remaining unexplained and which, in some cases, led to reduced power operation or reactor scrams [25-28].

In the area of Monte Carlo simulations, the main tools being developed within HPMC and McSAFE are highfidelity tools, which can also provide reference solutions to any low-order solution (e.g. nodal diffusion solvers) used by regulators and the industry in real life situations and for licensing purposes. Since the tools are able to provide unique full core solutions at the pin level taking into account local thermal hydraulic feedback, such tools substantially improve the modelling accuracy when predicting depletion and simulating static core configurations. In addition, the dynamic capability added to the Monte Carlo codes coupled with thermal hydraulic subchannel codes pave the way for the analysis of transients (e.g. REA, MSLB) with an unequalled accuracy as of today. Hence, these tools are very well suited for being used by the industry as a complement to low-order solutions. Finally, for all cases where no experimental data are available at a fine resolution, these tools can predict local safety-relevant parameters. With the maturity of the being developed Monte Carlo solutions, the project will allow industry-like problems to be modelled. This will provide a possibility to assess the adequacy of deterministic based solution methods that are routinely used by the industry and that rely on many approximations and limitations, as highlighted in Section 3.1.

The end users of the NURESIM software platform also benefit since the end of the project from the improvements made within the NURESAFE project in simulation capabilities, more precisely when e.g. they perform industrial studies, safety analyses, optimisation of reactor operation and reactor design. The end users are the members of the NURESAFE consortium (22 organisations) and the members of the NURESAFE Users' Group (five organisations). They can be categorized into (1) utilities (three utilities operating the majority of the European fleet of nuclear reactors), (2) one reactor and fuel manufacturer and vendor (Framatome), (3) three TSOs to safety authorities and (4) universities and research institutes. The standardised environment offered by the platform and the interoperability of codes facilitate collaborative work between all partners. Collaborative work contributes to the increase of the leadership of European science for nuclear reactor simulation.

Since the end of the NURESAFE project, further use and development of the software platform are pursued thanks to:

- a continuous maintenance by CEA of the software repository dedicated to the NURESIM platform;

- further development and maintenance of the generalpurpose software SALOME and URANIE (two opensource software supporting the entire platform);

- further development and maintenance of each individual software by code owners.

This above resulted in long-term frameworks that have already been used for many years.

\section{Conclusions and future recommendations}

Using the NURESIM platform, challenging DNS \& LES simulations were performed within NURESAFE to analyse bubbly flow with and without phase change in order to understand intricate phenomena that are beyond measurements capabilities. New modelling routes were proposed based on these results and were documented and implemented in the platform available to all stakeholders. Novel ideas were explored, and some others were further refined, such as combining large-scale and small-scale prediction techniques. Such techniques should in the medium term replace state-of-the-art methods that are limited to one flow regime. These novel techniques are applicable to more complex core-level thermal-hydraulic situations involving boiling. Solution procedures taking advantage of the coupling between various codes tackling different physics and scales were successfully developed.

In the area of Monte Carlo methods, the methods for depletion and dynamic calculations are close to their culmination. The developed coupled codes based on the ICoCo-methodology are now implemented in the European simulation platform NURESIM and the testing and validation phase will soon start. For this purpose, different benchmark problems of different size are being developed so that all partners will apply the developed tools for the analysis of those problems. Moreover, the validation of the codes under development using plant/ experimental data is of paramount importance for McSAFE. Therefore, plant data of two European reactors (PWR-KONVOI and VVER-1000) are being prepared and documented for the validation of the advanced depletion capability of the tools. On the other hand, selected SPERT III REA E test data will be used for the validation of the dynamic versions of the Monte Carlo codes. Finally, application to LWR and SMR are foreseen to demonstrate the extended capabilities of the multiphysics codes. Generally, it can be stated that considerable efforts are still needed for high-fidelity simulations based on Monte Carlo codes in an HPC-environment in order to perform core analysis with acceptable statistics for the key parameters of interest.

Beyond the major developments in computing capabilities for normal operation and design basis accidents, the monitoring of reactors and the early detection of anomalies will become increasingly important, due to the ageing fleet of reactors in Europe. By extending the current simulation platforms to the modelling of stationary fluctuations and their effect, such simulation tools can be used for creating large data sets that can thereafter be used to detect, from given measured reactor parameters, possible anomalies. For such a purpose, machine learning was demonstrated in CORTEX, using simulated test data, to be potentially capable of retrieving anomalies. Tests on actual plant data remain nevertheless to prove the viability of this technique. In addition, although the phenomena considered so far in CORTEX do not require taking the thermal-hydraulic feedback into account, the estimation of the coupled neutronics/thermal-hydraulics reactor transfer function might be necessary for other scenarios. 
In the area of neutron transport, it should also be noted that the methods being developed would allow modelling full core in pure transport. The limitations and approximations otherwise introduced when pre-generating assembly-wise macroscopic cross-sections would then be eliminated, thus greatly enhancing the level of faithfulness of neutron transport simulations for strongly heterogeneous cores (such as when using new fuel assembly designs, MOX fuel, etc.).

In essence, the different situations needing accurate modelling require the inclusion of more and more physics. Beyond neutronics, thermal-hydraulics and thermo-mechanics, other as important physics might need to be included: fuel physics, structural mechanics, coolant and radiation chemistry, radionuclide transport, etc. Truly multiphysics and multi-scale modelling approaches still need to be developed at a more mature level for tackling such situations. This includes the development of new models, their coupling, as well as the use of the latest advancements in numerical analysis optimized for HPC. In this respect, the development of hybrid methods, such as deterministic and probabilistic methods in neutron transport, or DNS, LES, CFD, and macroscopic approaches in fluid dynamics and heat transfer, should be favoured and optimized. This requires having different scientific communities collaborating and capitalizing on each other's strengths and expertise. With so challenging modelling targets, the use of machine learning for predictive modelling should also be considered, where machine learning could be used in place of or in addition to more traditional modelling approaches. The enormous amount of measured data at commercial reactors, research reactors, and experimental facilities represent a definite asset, in a machine learning-based modelling strategy that should be utilized as much as possible.

A complete list of the papers published within the projects can be found on the respective project websites: http://cortex-h2020.eu (for CORTEX), http://www. fp7-hpmc.eu (for HPMC), http://www.mcsafe-h2020. eu (McSAFE), http://www.nuresafe.eu (for NURESAFE).

\section{Nomenclature}

$\begin{array}{ll}\text { ATWS } & \text { Anticipated Transient without Scram } \\ \text { BWR } & \text { Boiling water reactor } \\ \text { CAD } & \text { Computer-aided design } \\ \text { CFD } & \text { Computational fluid dynamics } \\ \text { CNN } & \text { Convolutional neural network } \\ \text { CORTEX } & \text { CORe Monitoring Techniques and EXperi- } \\ & \text { mental Validation and Demonstration } \\ \text { DNS } & \text { Direct numerical simulation } \\ \text { EPFL } & \text { Ecole Polytechnique Fédérale de Lausanne } \\ \text { FEM } & \text { Finite Element Method } \\ \text { FSI } & \text { Fluid-structures interaction } \\ \text { HPC } & \text { High Performance Computing } \\ \text { HPMC } & \text { High Performance Monte Carlo Methods } \\ & \text { for Core Analysis }\end{array}$

HZDR Helmholtz-Zentrum Dresden-Rossendorf

LES

LSTM

$\mathrm{LWR}$

LOCA

McSAFE

Large eddy simulation

Long short-term memory

Light water reactor

Loss-of-coolant accident

MOX

MPI

MSLB

High Performance Monte Carlo Methods

for SAFEty Analysis

Main steam line break

NURESAFE NUclear REactor SAFEty Simulation Platform

NURESIM European Platform for Nuclear Reactor Simulations

NURISP NUclear Reactor Integrated Simulation

OpenMP $\quad$ Open multi-processing

PTS Pressurized thermal shock

PWR Pressurized water reactor

RAM Random access memory

REA Rod ejection accident

SIE Stochastic implicit euler

SMR Small modular reactor

SPERT Special Power Excursion Reactor Test Program

TSO Technical support organization

TUD Technical University of Dresden

UOX Uranium oxide

VVER Vodo-Vodyanoi Energetichesky reactor

\section{References}

1. C. Demazière et al., Overview of the CORTEX project, in Proceedings of the International Conference on the Physics of Reactors - Reactor Physics paving the way towards more efficient systems (PHYSOR2018), Cancun, Mexico, April 22-26, 2018

2. V. Sanchez et al., High performance Monte Carlo computing projects: from HPCM to McSAFE, in NUGENIA Forum, Ljubljana, Slovenia, April 14, 2015

3. L. Mercatali et al., McSAFE projects highlights, in International Multi Physics Validation Workshop, North Carolina State University, USA, June 2017

4. E. Deville, F. Perdu, Documentation of the Interface for Code Coupling: ICOCO, CEA, Paris, 2012

5. B. Chanaron et al., Advanced multi-physics simulation for reactor safety in the framework of the NURESAFE Project, Ann. Nucl. Energy 84, 166 (2015)

6. B. Chanaron, The European NURESAFE simulation project for reactor safety, in Proceedings of the Internationl Conferencre on the Nuclear Engineering (ICONE22), Prague, Czech Republic, July 7-11, 2014

7. C. Demazière, Multi-physics modelling of nuclear reactors: current practices in a nutshell, Int. J. Nucl. Energy Sci. Technol. 7, 288 (2013)

8. I. Lux, L. Koblinger, Monte Carlo particle transport methods: neutron and photons calculations (CRC Press, Boca Raton, FL, 1991) 
9. A. Ivanov et al., Internal multi-scale multi-physics coupled system for high fidelity simulation of light water reactors, Ann. Nucl. Energy 66, 104 (2014)

10. M. Däubler et al., High-fidelity coupled Monte Carlo neutron transport and thermal-hydraulic simulations using Serpent 2/SUBCHANFLOW - Part I: Implementation and solution verification, Ann. Nucl. Energy 83, 352 (2015)

11. F. Calivà, et al., A deep learning approach to anomaly detection in nuclear reactors, in Proceedings of the 2018 International Joint Conference on the Neural Networks (IJCNN2018), Rio de Janeiro, Brazil, July 8-13, 2018

12. F. De Sousa Ribeiro et al., Towards a deep unified framework for nuclear reactor perturbation analysis, in Proceedings of the IEEE Symposium Series on Computational Intelligence (SSCI 2018), Bengaluru, India, November 18-21, 2018

13. J. Dufek, J.E. Hoogenboom, Description of a stable scheme for steady-state coupled Monte Carlo-thermal-hydraulic calculations, Ann. Nucl. Energy 68, 1 (2014)

14. J.E. Hoogenboom, Demonstration of the time-dependence after a control rod movement, HPCM Deliverable D3.10 (2013)

15. J.E. Hoogenboom et al., Maximum efficiency in massively parallel execution of Monte Carlo criticality calculations, in Proceedings of the Joint International Conference on the Mathematics and Computation (MEC), Supercomputing in Nuclear Applications (SNA) and the Monte Carlo (MC) Method (MC2015), Nashville, USA, April 19-23, 2015

16. I. Mickus, J. Dufek, Optimal neutron population growth in accelerated Monte Carlo criticality calculations, Ann. Nucl. Energy 117, 297 (2018)

17. M. Garcia et al., Development of a spatial domain decomposition scheme for Monte Carlo neutron transport, in Proceedings of the International Conference on Nuclear Engineering (ICONE26), London, United Kindgom, July 22-26, 2018

18. D. Ferraro et al., Foreseen capabilities, bottlenecks identification and potential limitations of Serpent MC transport code in large-scale full 3D burnup calculations, in Proceedings of the International Conference on Nuclear Engineering (ICONE26), London, United Kindgom, July 22-26, 2018

19. M. Faucher et al., New kinetic simulation capabilities for TRIPOLI- $4^{\circledR}$ : methods and applications, Ann. Nucl. Energy 120, 74 (2018)

20. A. Livensky et al., Modeling the SPERT transients using Serpent2 with time-dependent capabilities, Ann. Nucl. Energy 125, 80 (2019)

21. S. Sanchez-Cervera et al., Optimization of multidimensional cross-section tables for few-group core calculations, Ann. Nucl. Energy 73, 387 (2014)

22. S. Lal et al., Direct numerical simulation of bubble dynamics in subcooled and near-saturated convective nucleate boiling, Int. J. Heat Fluid Flow 51, 16 (2015)

23. Y. Sato et al., Computational fluid dynamics simulation of single bubble dynamics in convective boiling flows, Multiphase Sci. Technol. 25, 287 (2013)

24. H. Li, H. Anglart, CFD model of diabatic annular two-phase flow using the Eulerian- Lagrangian approach. Ann. Nucl. Energy 77, 415 (2015)

25. Bundesamt für Strahlenschutz, Kurzbeschreibung und Bewertung der meldepflichtigen Ereignisse in Kernkraftwerken und Forschungsreaktoren der Bundesrepublik Deutschland im Zeitraum Januar 2011, Stand 14.12.2012 (2012) (in German)

26. Almaraz Trillo Report, Neutron noise status in Trillo NPP, Technical report CO-12/043, Spain, 2012

27. CSN/PDT/NCTRI/TRI/1503/203, Proposal of technical decision on the revision of the Technical Operational Specifications relative to the dead band of the filter in Trillo NPP, technical report (in Spanish)

28. M. Seidl et al., Review of the historic neutron noise behaviour in German KWU built PWRs, Prog. Nucl. Energy 85, 668 $(2015)$

Cite this article as: Christophe Demazière, Victor Hugo Sanchez-Espinoza, Bruno Chanaron, Advanced numerical simulation and modelling for reactor safety - contributions from the CORTEX, HPMC, McSAFE and NURESAFE projects, EPJ Nuclear Sci. Technol. 6, 42 (2020) 